\title{
Multi-keV X-ray Conversion Efficiency in Laser-Produced Plasmas
}

C.A. Back, J. Davis, J. Grun, L.J. Suter, O.L. Landen, J.H. Hammer, W.W. Hsing, and M.C. Miller

This article was submitted the $44^{\text {th }}$ Annual Meeting of the Division of Plasma Physics, Orlando, Florida, November 11-15, 2002

\section{October 31, 2002}

U.S. Department of Energy

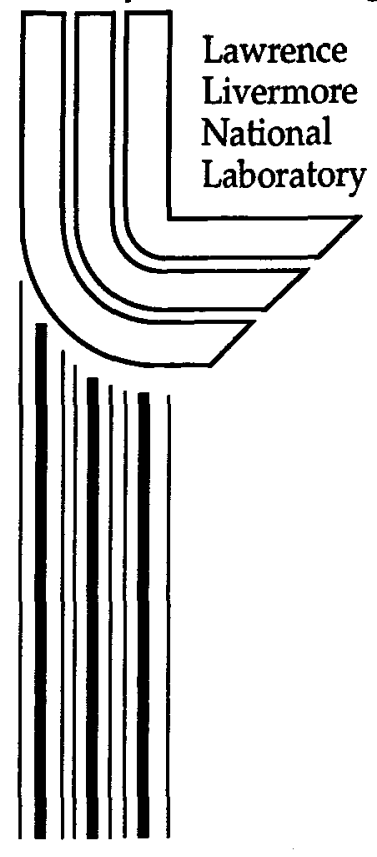




\section{DISCLAIMER}

This document was prepared as an account of work sponsored by an agency of the United States Government. Neither the United States Government nor the University of California nor any of their employees, makes any warranty, express or implied, or assumes any legal liability or responsibility for the accuracy, completeness, or usefulness of any information, apparatus, product, or process disclosed, or represents that its use would not infringe privately owned rights. Reference herein to any specific commercial product, process, or service by trade name, trademark, manufacturer, or otherwise, does not necessarily constitute or imply its endorsement, recommendation, or favoring by the United States Government or the University of California. The views and opinions of authors expressed herein do not necessarily state or reflect those of the United States Government or the University of California, and shall not be used for advertising or product endorsement purposes.

This is a preprint of a paper intended for publication in a journal or proceedings. Since changes may be made before publication, this preprint is made available with the understanding that it will not be cited or reproduced without the permission of the author.

This report has been reproduced directly from the best available copy.

Available electronically at http://www.doc.gov/bridge

Available for a processing fee to U.S. Department of Energy

And its contractors in paper from

U.S. Department of Energy

Office of Scientific and Technical Information

P.O. Box 62

Oak Ridge, TN 37831-0062

Telephone: (865) 576-8401

Facsimile: (865) 576-5728

E-mail: reports@adonis.osti.gov

Available for the sale to the public from

U.S. Department of Commerce

National Technical Information Service

5285 Port Royal Road

Springfield, VA 22161

Telephone: (800) 553-6847

Facsimile: (703) 605-6900

E-mail: orders@ntis.fedworld.gov

Online ordering: http://www.ntis.gov/ordering.htm

OR

Lawrence Livermore National Laboratory

Technical Information Department's Digital Library

http://www.llnl.gov/tid/Library.html 


\title{
Multi-keV X-ray Conversion Efficiency in Laser-Produced Plasmas
}

\author{
C. A. Back, J. Davis ${ }^{+}$, J. Grun \# , L. J. Suter, O. L. Landen, \\ J. H. Hammer, W. W. Hsing, and M. C. Miller
}

Lawrence Livermore National Laboratory, L- 21, P.O. Box 808, Livermore, CA 94551

\#Naval Research Laboratory, 4555 Overlook Ave, SW

Washington, DC 20375

${ }^{+}$Alme \& Associates, 6020 Richmond Hwy, Alexandria, VA 22303

Abstract

X-ray sources are created at the Nova and Omega laser by irradiating a confined volume of $\mathrm{Ar}, \mathrm{Xe}$, or $\mathrm{Kr}$ gas. The gas is heated by forty $0.35 \mu \mathrm{m}$ wavelength, 1-ns square laser beams to produce He-like ions that radiate K-shell emission over $\mathrm{mm}$-sized dimensions. The targets are designed to be "underdense", meaning that the initial gas density is lower than the critical density of the laser, $\mathrm{n}_{\mathrm{c}} \sim 10^{21} \mathrm{~cm}^{-3}$. The laser energy is primarily absorbed by inverse bremsstrahlung and a supersonic heat wave efficiently ionizes the gas. Results from time-resolved and time-integrated diagnostics over a range of experimental parameters are compared. This work represents an important, new method for development of efficient, large-area, tailored multi-keV x-ray sources.

\section{Introduction}

Gas-filled targets have been shown to achieve x-ray conversion efficiencies that are an order of magnitude greater than disk targets. ${ }^{1}$ They are being developed to use in radiography and material testing. Previous research at many international laboratories have embarked on $x$-ray conversion research because of its importance to diagnosing Inertial Confinement Fusion (ICF) plasmas and probing basic material structure. ( $\mathrm{ft}$ ) As higher power facilities such as the National Ignition Facility come on-line, more sophisticated future applications can require high photon energy, long temporal duration, and/or large size that current backlights cannot meet.

To satisfy upcoming challenges, we are exploring the characteristics of laserheated underdense targets. The goal is to enhance multi-keV $x$-ray output. 
Therefore, first and foremost, we need to create high temperature plasmas. Gasfilled targets allow us to do this because the initial density of the target is below the critical density of the laser, which enables a supersonic heating of the gas. Because important parameters governing the plasma evolution are different from those for disks, this research challenges our current understanding of non-LTE plasmas.

In this paper, we discuss the source characteristics of underdense radiators and present more of the supporting experimental data. First we will give an overview of previous $x$-ray source research and then discuss the physics of the underdense target heating. Because the dominant factors are not the same as for disks, the source characteristics impacting the multi-keV production are different. Characteristics addressed in this work include dependencies on the atomic number $Z$ and density.

An important figure of merit for $X$-ray sources is the conversion efficiency.

The conversion of laser energy into useable $x$-rays is quantified in conversion efficiency (CE) measurements where the total energy that is emitted by the source in a particular spectral bandwidth is divided by the absorbed laser energy. Laser-produced $x$-ray sources have been investigated largely in terms of the variation of laser parameters. For instance, papers have investigated $x$-ray conversion efficiency as a function of wavelength, pulse length, intensity, and focal spot. For solid disks, studies on with elements of different atomic numbers have produced an experimental database of conversion efficiencies. ${ }^{1-25} \mathrm{~A}$ compilation from many different laboratories show a steep decline in conversion efficiency with photon energy. Overall, the fraction emitted in multi-keV $x$-rays is $<1 \%$, even though the total conversion efficiency integrated over all photon energies may be significant, especially for higher Z elements. ${ }^{6}$

Many conversion efficiency studies have focused on the sub-keV x-ray range. ${ }^{7-9}$ Those studies have been motivated by ICF where near local thermodynamic equilibrium (near-LTE) radiation sources drive implosions. For ICF applications, the radiation drive is produced inside of hohlraums, cylindrical or spherical cavities that are irradiated by a laser. The frequency distribution of the source is nearly Planckian, peaking at $\sim 2.8 \mathrm{kT}$ and the energy is often characterized by $\sigma \mathrm{T}^{4}$ where $\sigma$ is the Stefan-Boltzmann coefficient and $\mathrm{T}$ is an effective blackbody radiation temperature.

Other studies have investigated conversion efficiency in the multi-keV $x$-ray range, mostly in order to quantify duration and intensity at specific photon energies useful for specific radiographic and material testing applications. At multi-keV energies, creating blackbody sources with frequency distributions that peak at multi-keV photon energies is not possible even with high-powered lasers. In fact, hohlraum designs tend to minimize multi-keV photons because they can be a source of preheat on the capsule which is undesirable. In most cases, the targets for multi-keV sources have been simple solid disks. Using these targets, current facilities cannot produces sources which have multi-keV conversion efficiencies of over $1 \%$. 
While much work has been done to understand the physics and optimize target design for sub-keV $\mathrm{x}$-rays, little work has been done to optimize backlight source output. Apart from studies as a function of laser parameters, the research to tailor multi-keV sources has largely been to fabricate mixed $\mathrm{Z}$ targets to increase the spectral bandwidth, or to use microdots or target angle to limit source size.(ft Zigler, me). The goal of this paper is to examine the underlying physics and to provide some useful scalings to help design suitable multi-keV sources.

\section{Underdense Sources}

Important characteristics of $x$-ray sources generally include the brightness, frequency distribution, temporal duration, size, and spatial uniformity. The frequency distribution and temporal duration of any particular source are determined by the application of interest. For inertial confinement fusion (ICF) sources have a near-blackbody frequency distribution, on the order of ns, and can either be constant in intensity, or shaped to optimize implosion trajectories. For radiography, maximum brightness in a limited bandwidth is needed with both long and short duration backlighting applications. The source size may be small to minimize spatial blurring in point projection applications, or, large for area backlighters. ${ }^{10,1}$

Underdense $x$-ray sources provide flexibility in design to address all of the important characteristics of $x$-ray sources discussed above. ${ }^{12}$ They are produced by laser-heating a gas which is confined in an enclosure. ${ }^{13,14}$ Ideally, the enclosure would be transparent to the $x$-rays of interest and define the volume of the radiator. It is filled with gas to a pressure such that the electron density in the plasma, $n_{e}$ is less than the critical density of the laser in $\mathrm{cm}^{-3}, \mathrm{n}_{\mathrm{c}}=1.1 \times 10^{21}$ / $\lambda_{L}{ }^{2}$, where $\lambda_{L}$ is the wavelength of the laser in microns.

Experiments reported here use Be "can" enclosures to confine the gas. They are identical to those reported before - they made from cylinders are $2 \mathrm{~mm}$ in diameter, $1.6 \mathrm{~mm}$ long, and $100 \mu \mathrm{m}$ thick which are capped with endcaps that have holes to allow the laser beam energy to irradiate the inside volume of gas.

The experiments were performed on the Nova and Omega $0.35 \mu \mathrm{m}$ wavelength lasers, using either $35.6 \pm 3.2 \mathrm{~kJ}$ in ten beams delivered in a $2 \mathrm{~ns}$ long square pulse or $20 \mathrm{~kJ} \pm 2.0 \mathrm{~kJ}$ in forty beams delivered in a $1 \mathrm{~ns}$ long square pulse, respectively. At both facilities, the beams remain at roughly the same power.

Five beams of Nova or 20 beams of Omega entered through laser entrance holes (LEH) from each side of a cylindrical Be "can", containing either Xe or Ar gas. At Nova, opposing beams produce overlapping spots on the inside wall. At Omega, 20 beams more effectively form a cone of irradiation. Schematics of the target and the laser irradiation are shown later in figures with $\mathrm{x}$-ray images.

In both cases, the laser beams were focused so that the expanding beams directly irradiated as large a volume of gas as possible. Best focus was placed outside the enclosure to keep beam intensities in a range of $2 \times 10^{15} \mathrm{~W} / \mathrm{cm}^{2}$ at the LEH to $4 \times 10^{14} \mathrm{~W} / \mathrm{cm}^{2}$ at the wall of the Be cylinder. The pathlength through the gas was $1.3 \mathrm{~mm}$ long from the LEH to the wall. 
LEH to $4 \times 10^{14} \mathrm{~W} / \mathrm{cm}^{2}$ at the wall of the Be cylinder. The pathlength through the gas was $1.3 \mathrm{~mm}$ long from the LEH to the wall.

The targets had gas fills of $80 \% \mathrm{Xe} / 20 \% \mathrm{Kr}, 100 \% \mathrm{Xe}$, or $100 \% \mathrm{Ar}$. The initial density of the plasma is measured with high accuracy by transducers which monitor the gas pressure. When ionized to a charge state of 44 , the nominal $1 \mathrm{~atm}$ gas pressure translates to $\mathrm{n}_{\mathrm{e}} \sim 1 \times 10^{21} \mathrm{~cm}^{-3}$, which is $\sim 0.1 \mathrm{n}_{\mathrm{c}}$. In the Xe experiments initial fill pressures varied from 0.5 to $2.3 \mathrm{~atm}\left(\sim 5-25 \% \mathrm{n}_{\mathrm{c}}\right)$. For the Ar, we have performed a scan of pressures ranging from 0.5 to $3.0 \mathrm{~atm}$ $\left(\sim 2-13 \% \mathrm{n}_{\mathrm{c}}\right)$.

Diagnostics are the same complement as described more fully before, however, the lines-of-sight are generally at 37.4 or 63.4 degrees on Omega whereas they are 0 or 90 degrees in the Nova experiments. These included timeintegrated crystal spectrometers, time-resolved x-ray diodes, and $x$-ray framing cameras. Specific diagnostic details are repeated in this paper when necessary.

To obtain Xe conversion efficiency measurements, the time-integrated spectrum is spectrally integrated over the $4-7 \mathrm{keV}$ energy range to obtain the total energy emitted in the L-shell radiation from the plasma with the assumption of isotropic emission. For Ar, the spectrum is integrated from 3-5 $\mathrm{keV}$.

\section{Comparison of a Disk vs. an Underdense Source}

Differences in plasma parameters are the basis for achieving higher multi-keV conversion efficiency. They can be revealed most clearly by comparisons between gas target to disk target simulations which do not introduce 3-D effects which can be difficult to model. For these purposes, the underdense radiator is a cylindrical gas column with a diameter equal to that of the laser focal spot. In Fig. 1, the temperature and density gradients for an underdense radiator initially at $0.01 \mathrm{~g} / \mathrm{cc}$ are compared to a denser disk target initially at $3.06 \mathrm{~g} / \mathrm{cc}$. In both figures, a $0.35 \mu \mathrm{m}$ wavelength laser with intensity $1 \times 10^{15} \mathrm{~W} / \mathrm{cm}^{2}$ is incident from the right onto a target at $\mathrm{z}=0$. Within the gas, laser absorption takes place predominantly by inverse bremsstrahlung at densities less than $n_{c}$. In the disk, electron conduction efficiently conducts laser energy deposited at the critical density into much denser and cooler regions of the disk. Multi-keV emission is typically created in high temperature regions where the heated material becomes highly ionized. Thus, for disk targets in which the density steeply falls as the plasma ablates off the surface of the target in the positive $z$-direction, a small volume of the plasma is at the optimal plasma conditions for multi-keV production. For an underdense radiator where the laser has propagated $\sim 1 \mathrm{~mm}$ into the gas, the volume where $n_{e}>10^{21} \mathrm{~cm}^{-3}$ and temperatures $>5 \mathrm{keV}$ has significantly increased.

The calculated overall energy balance is very different because of the method by which the plasma is formed. The supersonic heating of an underdense gas basically eliminates the ablative subsonic heating that occurs in solid disk targets. 
In Fig. 2, we graph the absorbed laser energy partitioned into electron thermal energy, kinetic energy, and radiation $<4 \mathrm{keV}$, and radiation $>4 \mathrm{keV}$. The ion kinetic energy is negligible for the two targets.

The kinetic energy is given by $1.5 \mathrm{~m}_{\mathrm{e}} \mathrm{v}_{\mathrm{e}}^{2}+1.5 \mathrm{~m}_{\mathrm{i}} \mathrm{v}_{\mathrm{i}}^{2}$ while the thermal energy of the plasma is $1.5 \mathrm{n}_{\mathrm{e}} \mathrm{kT}$ er , $1.5 \mathrm{n}_{\mathrm{e}} \mathrm{kT}$ for the electron or ions, respectively. From these calculations, we find that gas-filled targets are much more efficient at increasing the temperature in the plasma, thereby increasing the thermal energy in the plasma at the expense of the kinetic energy of the plasma. The higher temperature means that more energy is expended ionizing and heating the gas.

The total radiated energy is the sum of the fractions of radiation above and below $4 \mathrm{keV}$ in Fig. 2 . Although the total radiated energy is slightly larger in the disk target, this is deceptive since the majority is emitted as sub-keV $\mathrm{x}$-rays generated in the denser ablative part of the target. The reduction in soft (sub$\mathrm{keV}) \mathrm{x}$-ray emission can be seen in Fig. 3 where the emission as a function of photon energy is plotted for the Xe-gas and a Xe disk.

The spectra shown in Fig. 3 are calculated using an average atom model. Lshell emission is complex; therefore simplified atomic models are often used in calculating the emission in hydrodynamic simulations. These codes are not spectroscopically accurate. However to first order, the energy balance in the target is insensitive to radiation losses, so the trend in the relative weightings of the sub-keV and multi-keV energies are meaningful. Calculations of the $\mathrm{x}$-ray using a more detailed atomic model can produce $\sim 25 \%$ differences in absolute flux [16]. For the Xe disk, the high density plasma in the conversion layer is primarily heated by conduction and its radiation peaks near $1 \mathrm{keV}$. Calculations of the gas-filled target show that the emission of the L-shell at $\sim 4.8 \mathrm{keV}$ dominates the sub-keV emission. Therefore, the fraction of thermal energy in the target, which is associated with the low temperature, sub-keV emission, is smaller. For plasma conditions at $5 \mathrm{keV}$ in the plasma emission can be approximated by a coronal model because its temperature is in the range of 1 to 3 times the ionization energy of the dominant ions in emission, $4.8-5.4 \mathrm{keV}$ for Lshell Xe.

\section{Dominant Physical Processes in the Plasma}

In the previous paper, we give analytic equations which describe the heating and cooling processes occurring in the source. Here we revisit them in order to provide a more fully illustrate essential aspects of the source.

The target is heated by the absorption of laser light through collisional damping of the laser light wave. During this time, a heat front propagates supersonically through the target. The absorption length over which energy is deposited is determined by the inverse bremsstrahlung length, $\ell$, where $Z$ is the ionic charge (neutral $=0$ ), $\ln \Lambda$ is the Coulomb logarithm, $\lambda_{L}$ is the laser 
wavelength in $\mu \mathrm{m}, \mathrm{T}_{\mathrm{e}}$ is the electron temperature in $\mathrm{keV}, \mathrm{n}_{\mathrm{e}}$ is the electron density in $\mathrm{cm}^{-3}$, and $\mathrm{n}_{\mathrm{c}}$ is the critical density in $\mathrm{cm}^{-3} .{ }^{16}$

$$
\ell=\frac{0.56 \lambda_{L}^{2} T_{e}^{3 / 2}}{\left(n_{e} / n_{c}\right)^{2} Z \ln (\Lambda)} \sqrt{1-\left(n_{e} / n_{c}\right)}
$$

For Xe-filled targets discussed below, plasma conditions produce an average $\mathrm{Z}=44, \mathrm{n}_{\mathrm{e}}=0.1 \mathrm{n}_{\mathrm{c}^{\prime}}$ and $\mathrm{T}_{\mathrm{e}} \sim 5 \mathrm{keV}$. For a Coulomb logarithm In $\Lambda=8$, the absorption length is $\sim 0.5 \mathrm{~mm}$. The inverse bremsstrahlung absorption length determines how much plasma can be heated with a laser facility of a given energy. For our current lasers, this equation shows that mm-size plasmas can be created.

Cooling, on the other hand, is due to electron conduction and radiation as discussed in the previous paper. The description of the electron conduction losses are dependent on the surface area of the heated volume. The maximum area for conductive cooling is conveniently defined by the outer structure of the Be "can", thus even with some hydrodynamic evolution, a simple model is still seen to remains dependent on temperature. Coronal models of a radiatively cooling plasma show somewhat complex dependence on $\mathrm{T}$ when the plasma is ionized high enough to emit L-shell radiation. However it is proportional to the density squared. The power lost from these competing rates is plotted, in Fig. 4. It shows that if the density increases by a factor of only 2 , there can be a significance enhancement of the L-shell radiation.

\section{X-ray Imaging}

In this section we will concentrate on the data provided by the $x$-ray images. Time-resolved $x$-ray framing cameras provide pinhole images of the source that are integrated over $\sim 80 \mathrm{ps}$. With these images, we can provide evidence that the physical mechanisms described above are manifested in $x$-ray images of the source size. Furthermore, 2-D images also helped provide a means for optimizing the conversion efficiency.

Fig. 5 shows a sequence of imaging data from Nova experiments which have a 5-fold symmetry around the "can" axis. The bleaching wave formed by the laser creates a high temperature sufficient to ionize Xe to L-shell in the plasma quickly. L-shell emission begins instantaneously and monotonically grows and peaks near the end of the pulse. 2-D images show the entire volume emitting 1 ns into the $2 \mathrm{~ns}$ pulse for photon energies greater than $4 \mathrm{keV}$. The Nova experiments were performed with the $\mathrm{Xe} / \mathrm{Kr}$ gas mixture, and images at photon 
energies greater than $13 \mathrm{keV}$ from $\mathrm{Kr}$ do show spatial variations related to the plasma heating, indicating that either the temperature or density were not uniform in the target.

The spectra and diode data have been discussed in the previous paper. Of particular note is that the Bragg crystal spectra were used to determine the experimental $\mathrm{T}_{\mathbf{e}}$. We measure a time-averaged plasma electron temperature in the range of 4.5 to $5.5 \mathrm{keV}$. This measurement provides assurance that the inverse bremsstrahlung length is adequate to heat the target.

The first confirmation that inverse bremsstrahlung is effective is found in the fact that the $x$-ray emission begins at the onset of the heating laser pulse. Furthermore, in the images at $1.3 \mathrm{~ns}$, the entire volume of the target is in emission an individual images of the laser beams are not identifiable. We find that the target can be uniform to at least $10 \%$ and larger variations in other shots can be correlated to more poor beam energy balance. This provides evidence that the electron conduction in the non-irradiated areas is sufficient to heat the plasma to at least $4 \mathrm{keV}$, otherwise, the L-shell emission would not be observed.

The brighter emission at the wall, particularly apparent in the axial view, is also of interest. The wall is made of Be and should not emit more than the heated gas at these photon energies. The Be wall material is provided by Brush Wellman and the material certification shows that the material is were $99.39 \%$ Be. (P.O. \# 1448403), where the contaminants are largely low to mid-Z materials. The highest $\mathrm{Z}$ contaminant is $\mathrm{Ti}$ at 0.004 $\%$ which is a small enough percentage to have a negligible effect on the emission, even if $\mathrm{Ti} \mathrm{He} \alpha$ lines at $4.7 \mathrm{keV}$ are produced. Independent target characterization on site at LLNL of the actual target shows impurities of $\mathrm{O}, \mathrm{Al}, \mathrm{Si}, \mathrm{S}, \mathrm{Cl}, \mathrm{K}, \mathrm{Ca}$ and $\mathrm{Fe}$ in small quantities that total less than $0.1 \%$ by weight. Again this confirms that the impurities are insufficient to cause significant amounts of emission in these target images. Thus, the higher emission observed is probably an effect due to the higher density of the gas near at the wall since the Be is expected to ablate towards the center of the hohlraum and thus compress the gas.

Fig. 6 shows $x$-ray images of the target from the side view filtered for two different photon energy bands. The lasers enter the hohlraum from the left and right of the target. On the left is an image filtered for $x$-rays at $4 \mathrm{keV}$ and above. Here it is fairly uniform in the second half of the pulse and some plasma is seen escaping the laser entrance holes. On the right in $x$-ray images filtered for $>13 \mathrm{keV}$ a two lobed emission pattern is still visible early in time, but at about $1.5 \mathrm{~ns}$, shown on the right, it remains non-uniform even later in the pulse. The data shows that the lasers have already propagated into the center of the hohlraum by $1 \mathrm{~ns}$ for $\mathrm{Xe}$ and $\sim 1.3 \mathrm{~ns} \mathrm{Kr}$.

Optimization of the target geometry was largely done experimentally. Simulations predict that approximately $90 \%$ of the laser energy is absorbed in the plasma. We are unable to measure transmission through the Be wall. However, the non-uniformity of the spatially-resolved images provided guidance to improving the conversion efficiency. Initial experiments produced X-ray images that implied that the laser energy was not propagating into the "can" as expected because the central volume of gas was never observed in emission. Detailed post-simulations found that backscatter and inhibited electron conduction could not account for the unheated areas of the target. Further modeling with improved resolution at the LEH suggested that refraction of the laser 
beams was occurring in the low density Be plasma formed at the edges of the entrance holes. To test this hypothesis, the LEHs were increased from $1.0 \mathrm{~mm}$ to $1.5 \mathrm{~mm}$ in diameter. This led to the fairly uniform $x$-ray images shown here and also shifted the peak of the x-ray pulse to the end of the laser pulse. Calculations with refraction were also able to reproduce the experimentally measured temporal emission, leading us to conclude that refraction was no longer a problem.

An indication of the limits of the electron conduction are seen in Fig. 7 where a larger $3.6 \mathrm{~mm}$ diameter Be "can" was used. The predictions by large scale simulations were that the conversion efficiency would be comparable, but in fact it was only about $80 \%$ of the smaller $2 \mathrm{~mm}$ diameter targets. The x-ray images for these larger targets show the presence of individual plasma columns in emission in the area where the beam directly irradiates the gas. Images taken end-on from the axial pinhole imager are shown for the small and large "cans". The blue and red circles in Fig 6 and 7show the diameter of the two different sized cansiviewed end-on. The smaller diameter target images show bright emission at the wall of the $2 \mathrm{~mm}$ diameter can which corresponds to the position of the incoming beams on the inside wall of the hohlraum.

Fig. 8 shows the temporal history of the x-ray pulse of the L-shell of Xe. The red curve is the new data showing a longer duration of the $x$-rays of interest. The green curve is for the old design with a smaller LEH and a $1 \mathrm{~atm}$ fill and the blue curve is for the larger $3.6 \mathrm{~mm}$ diameter target. Based on the evolution of the images, we believe the rise in the signal is correlated with the increasing volume of plasma in emission. The fall of the Xe temporal signal is dependent on the simple cooling of the laser-heated volume. According to calculations, the radiative cooling after the end of the laser pulse occurs primarily in the sub-keV range.

At Omega, the irradiation of the gas-filled target is much more axially symmetric since 20 beams enter from one side. These are taken at an angle of 63.4 degrees from the "can" axis. These images are taken over the $1 \mathrm{~ns}$ pulse. This is much more similar to the calculational geometry. Comparable images for a $0.5,1.0$, and $2.2 \mathrm{~atm}$ Xe target taken at Omega are shown together. The enhanced filling produces an image that is qualitatively more similar to calculations that tend to show two lobes of emission.

Data from different targets at Omega taken for pressures corresponding to 1021 clearly show the atomic number dependence for the inverse bremsstrahlung. Fig. 10 shows that Ar gas target, having a lower $Z$, is observed in emission over the full volume of the Be "can". However, the Xe gas target shows two distinct lobes of emission. The central volume of the plasma never heats up.

CALCULATIONAL DETAILS OF IMAGES - EMISSIVITY, te ne- comp $\mathrm{w} /$ codes 


\section{Multi-keV Conversion Efficiency}

Since L-shell conversion efficiencies have not been as extensively investigated as K-shell or M-shell conversion efficiencies, we undertook the comparison study of the slab conversion efficiency with beams spatially smoothed using KPP phase plates. These CsI experiments were performed with the same diagnostics as those used for the Xe-filled Be cans. They were performed by irradiating a slab target which had $6 \mu \mathrm{m}$ of CsI coated on each side.

Past research has been difficult to reconcile with simulations because the experimental laser intensity was difficult to model. Due to limited energy available at laser facilities, the laser intensity was varied by changing the spot size. Also, although beam image relaying techniques improve the spatial beam profile, the actual spatial intensity profile still contains hot spots unique to each beam.

In this study we improve both of these experimental limitations. KPP phase plates [19] were used to smooth the beams by eliminating $>10 \mu \mathrm{m}$-sized hot spots in the spatial profile and to define a $400 \mu \mathrm{m}$ FWHM spot for each individual beam on target. The dependence of the conversion efficiency on laser intensity was investigated by varying the laser energy for a given spot size. The dependence of the conversion efficiency on source size was experimentally investigated by varying the overlap of the beams. The dependence of the conversion efficiency on laser intensity was investigated by varying the laser energy for a given spot size.

Since simulations typically employ a smooth envelope to model the spatial distribution of the laser beam intensity and not the far field intensity profile of the beam itself, these solid disk measurements discussed here provide a cleaner comparison to simulations than the typical reported data of this kind.

For the CsI solid target experiments, Fig. 11 show a plot of conversion efficiency as a function of laser intensity. We also show predictions from calculations of these experiments. X-ray conversion efficiencies are 0.8 to $1.7 \%$ were obtained from these experiments. They are lower than initial predictions by at least a factor of 2 . Calculations show that there is a significant dependence on the flux limiter and possible B-field effects that affect the simulated conversion efficiencies and this is being investigated. Two dimensional modeling of the expansion also affects these calculations, generally lowering the conversion efficiency due to expansion of the plasma. Calculations using two different flux limiters, $\Phi$, in two-dimensional simulations give significantly different results. These flux limiters span a reasonable range for modeling ICF targets [20]. Physically, a higher flux limiter corresponds to a longer length of conduction region. These simulations are over a factor of two lower than the one-dimensional simulations. However, the experimental results are still below the predictions from two-dimensional simulations. 
Finally, in Fig. 12 we show a plot of the conversion efficiency versus electron density or pressure. The point for a solid target, CsI, at a density of $\mathrm{N}_{\mathrm{e}} \sim 4 \times 10^{23}$ is also placed on the graph in order the represent the conversion efficiency of an ablative target on the same graph. It is clear from the data that the Xe conversion efficiency peaks near critical density. We are unable to pressurize targets higher to obtain higher density points due to the fragility of the $\mathrm{CH}$ windows.

However, based on our understanding of the sources, it is reasonable to conclude that we are near the peak of the optimization in pressure.

\section{NIF-scaling}

As discussed in the previous paper, the primary heating during the laser pulse can be estimated as a power balance between the heating by the incoming laser energy and the cooling due to multi-keV radiation and electron conduction into the gas surrounding the laser-heated volume. Figure 13 shows the calculations and the scaling of multi-keV radiators to NIF laser powers. It agrees fairly well for calculations on a spherical gas-filled target. The $x$-ray power and intensity both scale up with laser power.

More research will investigate the physical processes in more detail to enable better tailoring of the gas-fill and target sizes. However, since the gas heating is largely governed by the inverse bremsstrahlung absorption, we can develop a scaling which allows us to predict $x$-ray conversion efficiencies that should be realizable on the National Ignition Facility.

\section{Conclusions}

New multi-keV sources have been developed on the Nova laser. These sources are underdense radiators that take advantage of bound-bound radiation to maximize emission in the $4-7 \mathrm{keV}$ photon range. The method of production is by supersonic heating of a confined gas. This technique allows efficient ionization of high- $Z$ atoms, with less energy expended in kinetic energy and parasitic sub-keV emission losses.

The high conversion efficiency of these targets is due to optimization of the initial target conditions and laser parameters to produce high temperature plasmas. Laser energy coupling into the target is still important. However, as long as initial conditions are controlled to ensure supersonic heating with minimum backscatter, future advanced designs for gas-filled targets can be more focused on design of the target, i.e. the choice of gas, and use of hydrodynamic motion to maximize the emission. Laser-produced plasma sources have advantages over other $\mathrm{x}$-ray sources because the target design and laser irradiation conditions can be optimized to create the source.

The radiators have well-defined, uniform source size which is advantageous for radiograph, material testing, and next-generation fluorescence-type diagnostics. Furthermore, applications of these types of sources can be very diverse. For instance, the targets are modular and for future NIF applications, they can be multiplexed to provide extended sources for irradiating large areas. 
Or with standardized laser and target conditions, they may become instrument calibration sources. These studies are on gas-filled targets which have recently shown promise as new backlight sources because they scale more favorably to higher photon energies.

\section{ACKNOWLEDGEMENTS}

The authors thank R. Kauffman for discussions and F. Ze for crystal calibrations. This work was performed under the auspices of the U.S. Department of Energy by University of California Lawrence Livermore National Laboratory under contract No. W-7405-Eng-48 and is supported in part by the U. S. DTRA contract number IACRO 98-3064, Work Units 57424 and 57425.

\section{FIGURES ;}

Figure 1 One-dimensional calculations comparing the plasma parameters for a cryogenic Xe disk versus a Xe gas column irradiated by a laser.

Figure 2 Energy partition in a plasma created from a cryogenic Xe disk and a Xe gas target.

Figure 3 Average atom non-LTE spectra calculated for disk and gas target plasmas.

Figure 4 Cooling rates plotted for electron conduction and L-shell radiation

Figure 5 Series of $x$-ray images of the L-shell emission from a Xe-filled Be can irradiated at the Nova laser facility. A schematic of the target and laser irradiation shows the target as seen by the diagnostic.

Figure 6 Two-dimensional x-ray images of the Nova target. The image of the left is for energies $>4 \mathrm{keV}$ and is dominated by the $\mathrm{Xe}$ emission at $1.3 \mathrm{~ns}$. The image on the right is filtered for energies $>13 \mathrm{keV}$ at $1.3 \mathrm{~ns}$ and are primarily from $\mathrm{Kr}$ in the gas target.

Figure 7 Images of $3.6 \mathrm{~mm}$ diameter target. $X$-ray image for a $1 \mathrm{~atm}$ pressure target at photon energies $h v>4 \mathrm{keV}$ at $1.8 \mathrm{~ns}$ into the pulse. In the $\mathrm{Xe}$ image, the larger volume is not uniform and the corners of the target are not heated sufficiently to emit $>4 \mathrm{keV} x$-rays. In the $\mathrm{Kr}$ image, the emission is in the center and does not spread to the full diameter of the can.

Figure 8 Temporal diode traces of Xe target 
Figure $9 \mathrm{X}$-ray images of Xe targets for pressures of 0.5, 1.0 and 2.2 atm. The schematic of the laser configuration at Omega is roughly tilted to be at approximately the same angle as the data. However, the image is a project of the target viewed $63.4^{\circ}$ from the cylindrical axis.

Figure 10 Xe vs Ar images, both at about $0.8 \mathrm{~ns}$ for targets of $\mathrm{Ne} \sim 1 \times 10^{21} \mathrm{~cm}^{-3}$

Figure 11 CsI conversion efficiency as a function of laser intensity. Two 2dimensional simulations use different flux-limiter values as discussed in the text.

Figure 12 Multi-keV x-ray conversion efficiency of Xe vs electron density

Figure 13 Summary of multi-keV $x$-ray conversion efficiencies. The Te scaling provides a useful scaling of the conversion efficiencies with laser power.

\section{REFERENCES}

1 C. A. Back, J. Grun, C. Decker, et al., Phys. Rev. Lett. (USA) 87, 275003/1 (2001).

1 . Matthews, D. L., Campbell, E. M., Ceglio, N. M., et al., J. Appl. Phys. 54,. 4260 (1983).

3 . Phillion, D. and Hailey, C. J., Phys. Rev. A 34, 886 (1986).

4 Kodama, R., Okada, K., Ikeada, N., et al., J. Appl. Phys. 59, 3050 (1986).

s. Yaakobi, B., Bourke, P., McCrory, R., et al, Opt. Commun. 38, 196 (1981).

'. Kauffman, R. L., "X-ray Radiation from Laser Plasma" in Handbook of Plasma Physics, vol. 3, eds. Rubenchik and Witkowski, pp. 111-162 (Elsevier Science, North-Holland, 1991) and references therein.

7. Sigel, R., Eidmann, K. Lavarenne, F. and Schmalz, R. F. Phys. Fluids B 2, 199 (1989); Eidmann, K., Schmalz, R. F. and Sigel, R. Phys. Fluids B 2, 208 (1989).

8. Mochizuki, T., Yabe, T. , Okada, K., Hamada, M., Ikeda, N., Ikyokama, S. and Yamanaka, C., Phys. Rev. A 33, 525 (1986).

9. Kauffman, R. L., Suter, L. J., Darrow, C. B., Kilkenny, J. D., et al., Phys. Rev. Lett. 73, 2320 (1994).

10 . Glendinning, S. G., et al., Phys. Plasmas 7, 2033 (2000); ); Ze, F., et al., J. Appl. Phys. 66, 1937 (1989).

${ }^{11}$. Lindl, J. D., Phys. of Plasmas 2, 3933-4024 (1995) and references therein.

${ }^{12}$. C. A. Back, J. Grun, C. D. Decker, J. L. Davis, O. L. Landen, L. J. Suter, and R. Wallace, " Production of multikilovolt X-rays from laser-heated targets", Applications of X Rays Generated from lasers and Other Bright Sources, ed G. A. Kyrala and J-C Gauthier, 3157, 130-137, SPIE, Bellingham, WA (1997).

${ }^{13}$. Kauffman, R. L., et al. ICF Quarterly Report 6 (2), 96, Lawrence Livermore National Laboratory, CA UCRL-LR50021-96-2 (1996).

14. Suter, L. J., Kauffman, R. L., Davis, J. F. and Maxon, M. S., ICF Quarterly Report 6 (3), 96, Lawrence Livermore National Laboratory, CA UCRL-LR-105821-96-3 (1996).

15. C. Decker, private communication.

${ }^{16}$. Dawson, J., Kaw, P. , and Green, B. , Phys. Fluids 12, 875 (1969); Lindl, J. Inertial Confinement Fusion, SpringerVerlag, New York, 1998, chapter 8 and 11.

17. Dixit, S. N., Feit, M. D., Perry M. D. and Powell, H. T., Opt. Lett. 21 , 1715 (1996).

${ }^{18}$. Fabbro, R. Max, C., and Fabre, E., Phys. Fluids 28, 1463 (1985). 

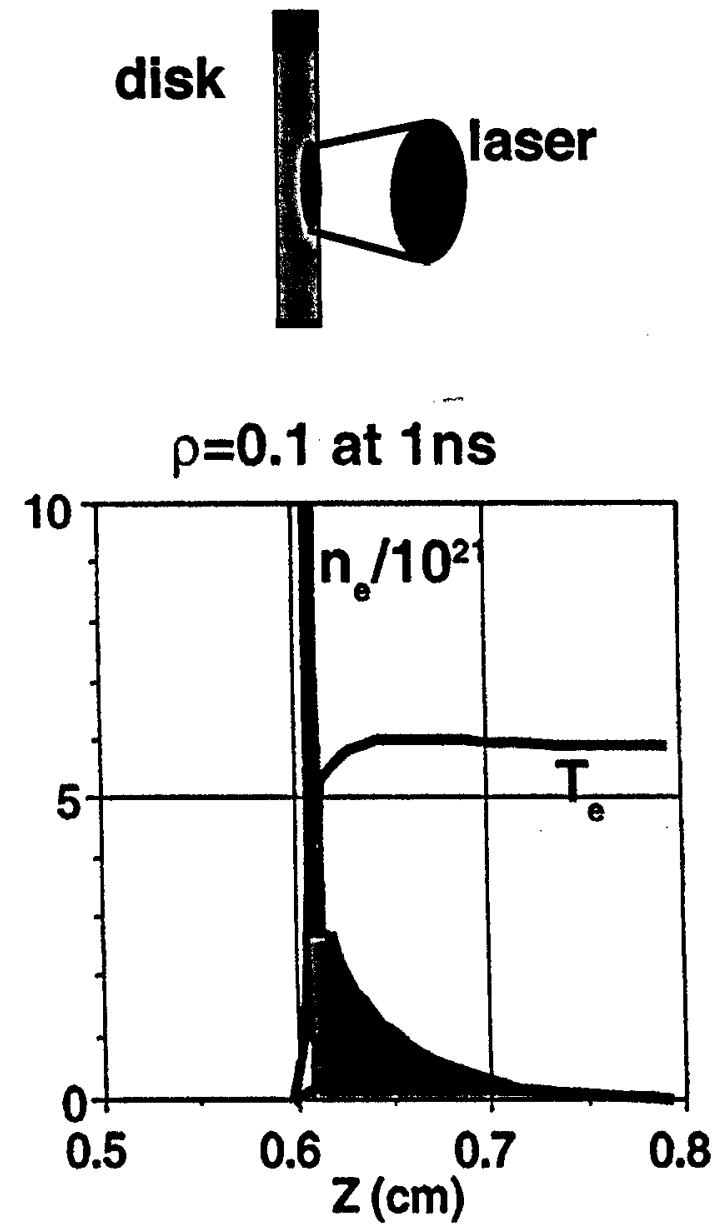
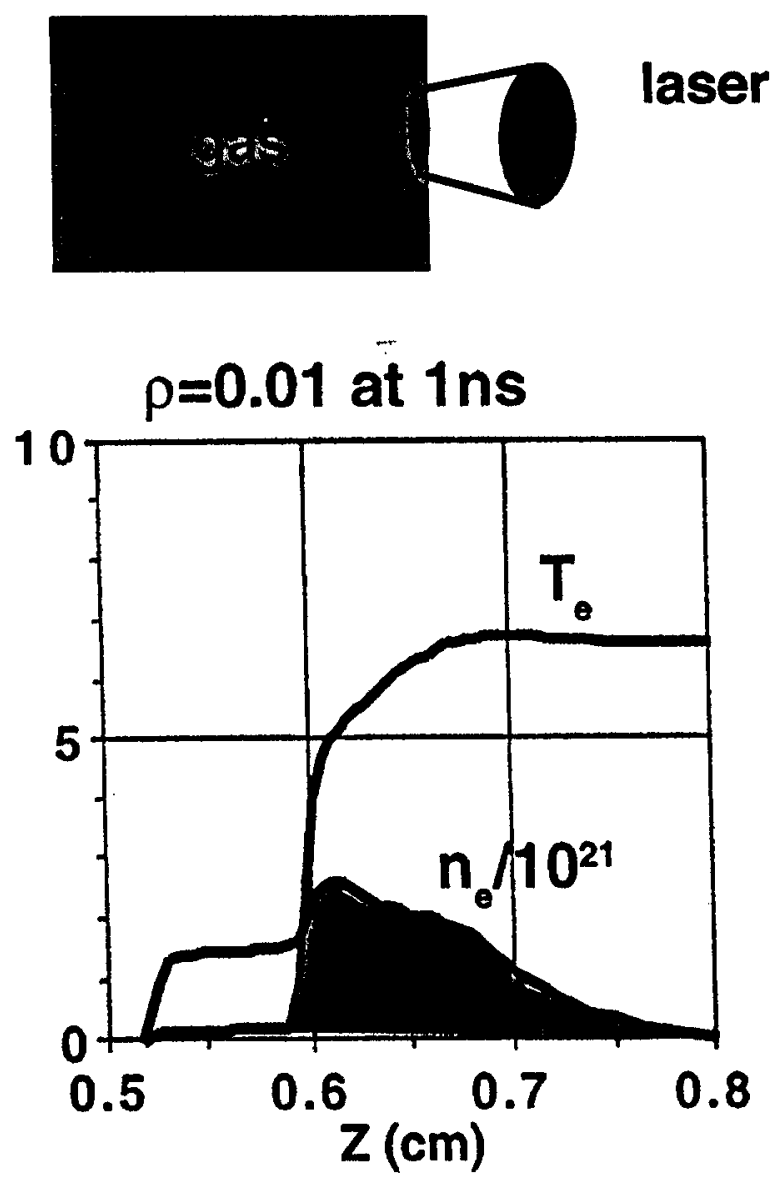

Fig.1

POP

Back, et al. 
Energy Budget at 2ns

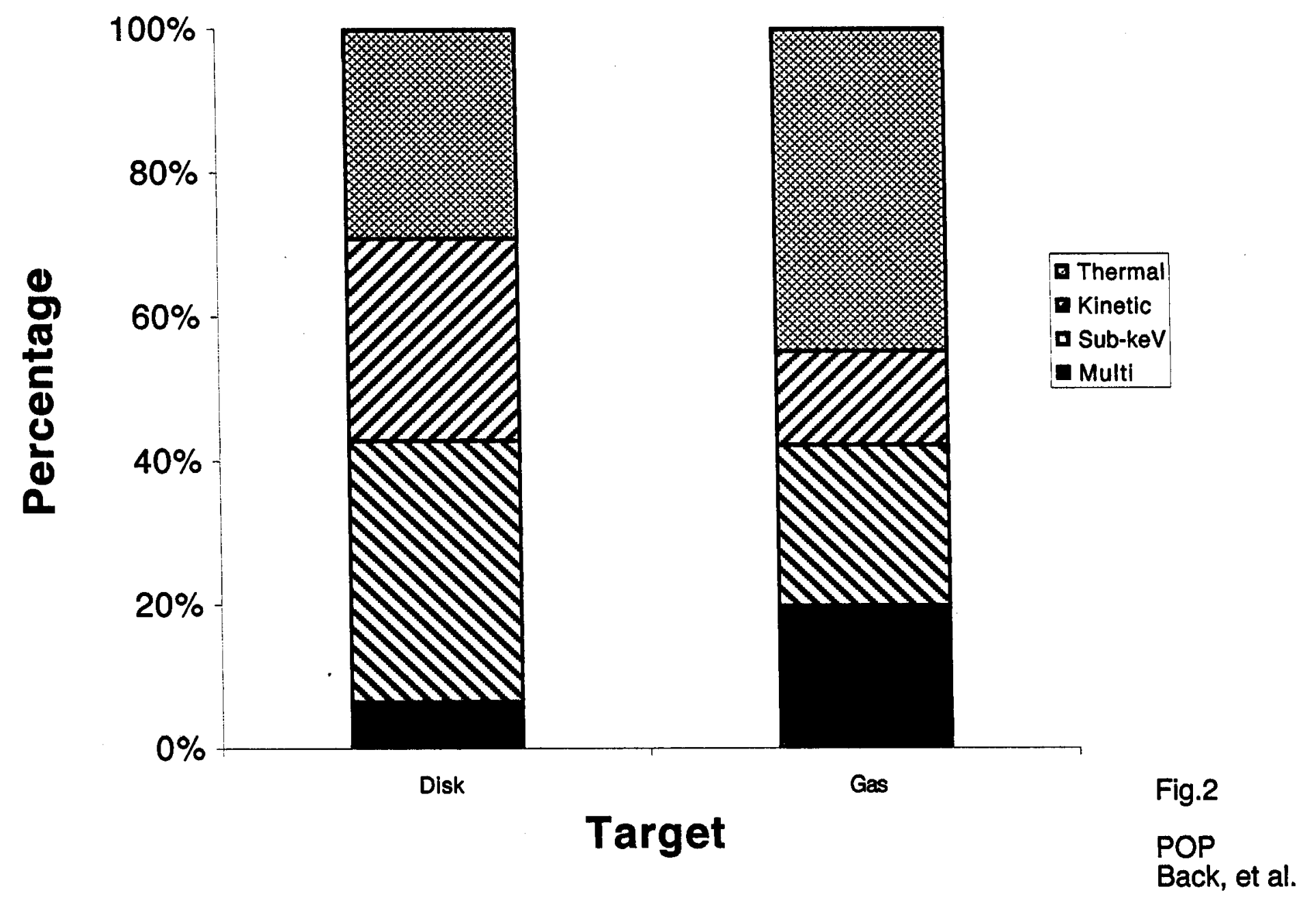



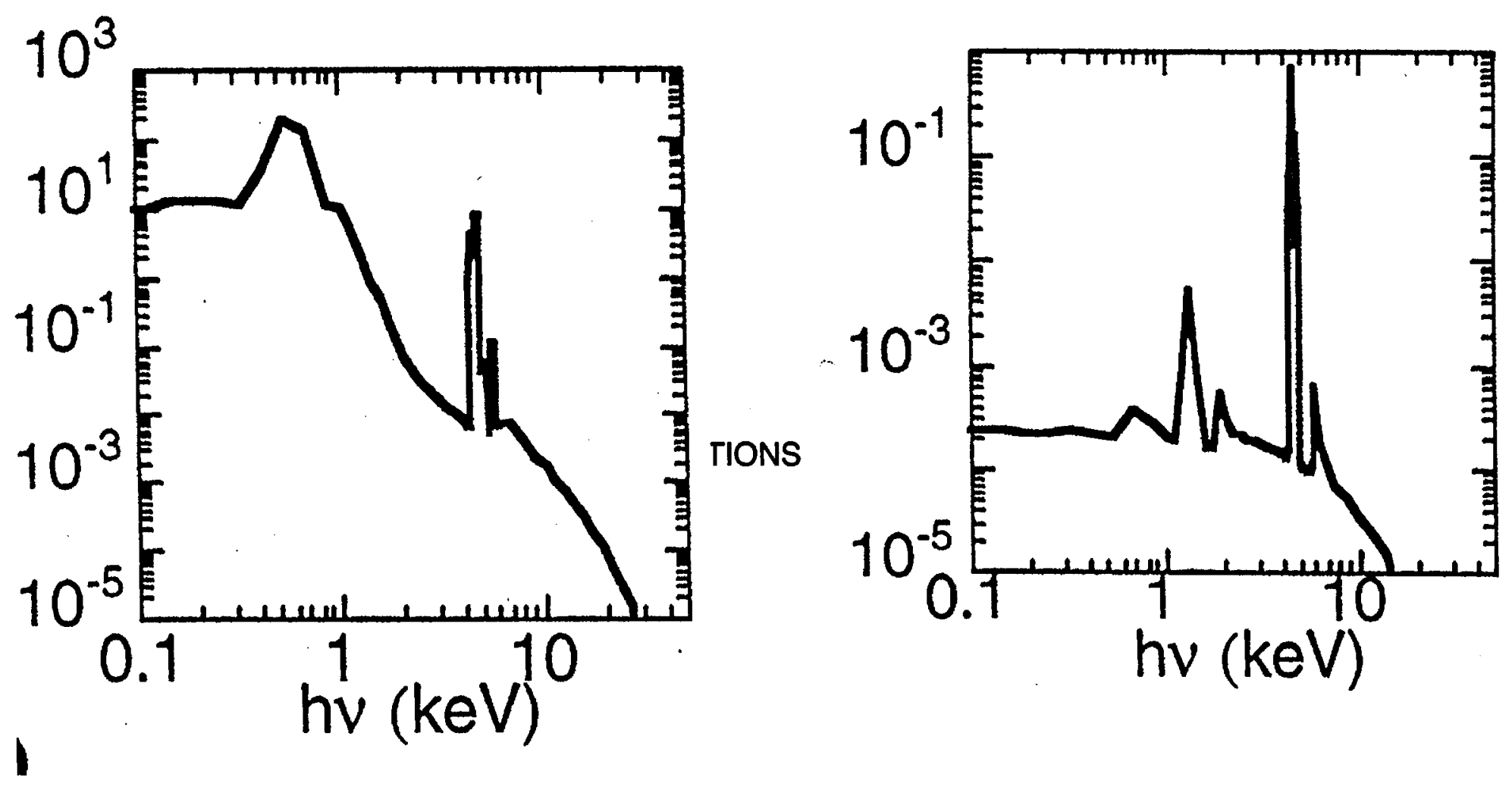

Fig. 3

POP

Back, et al. 


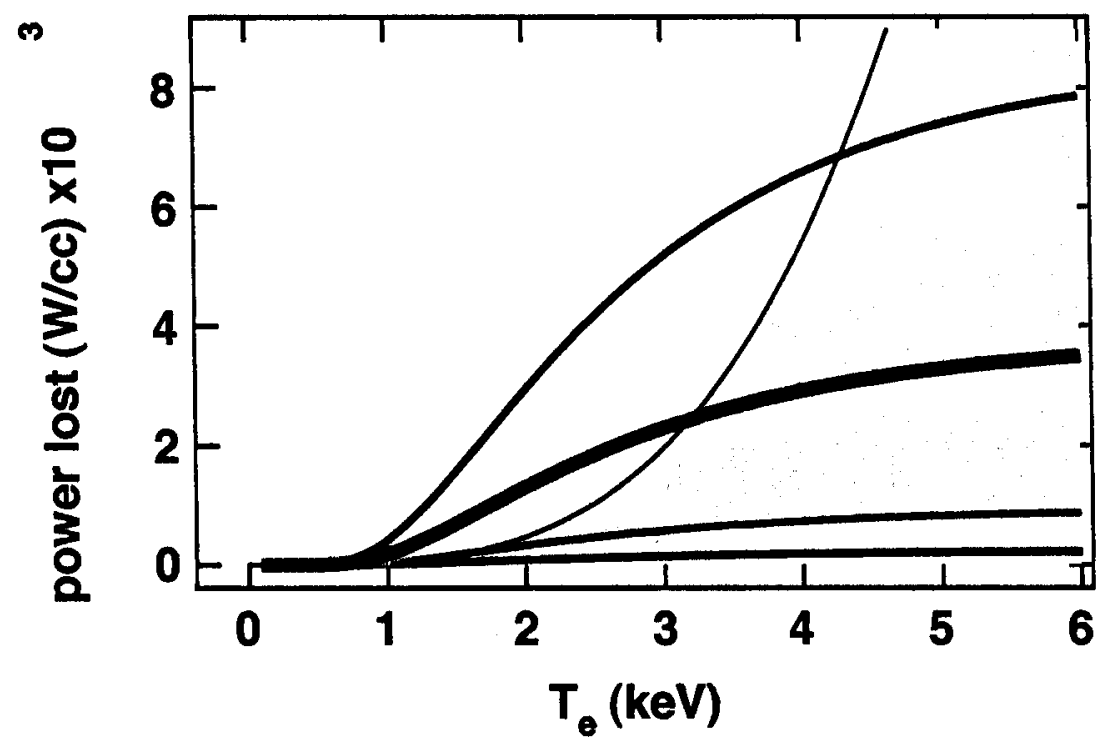

Fig.4

POP

Back, et al. 


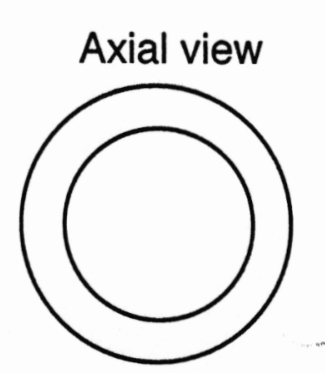

Side view
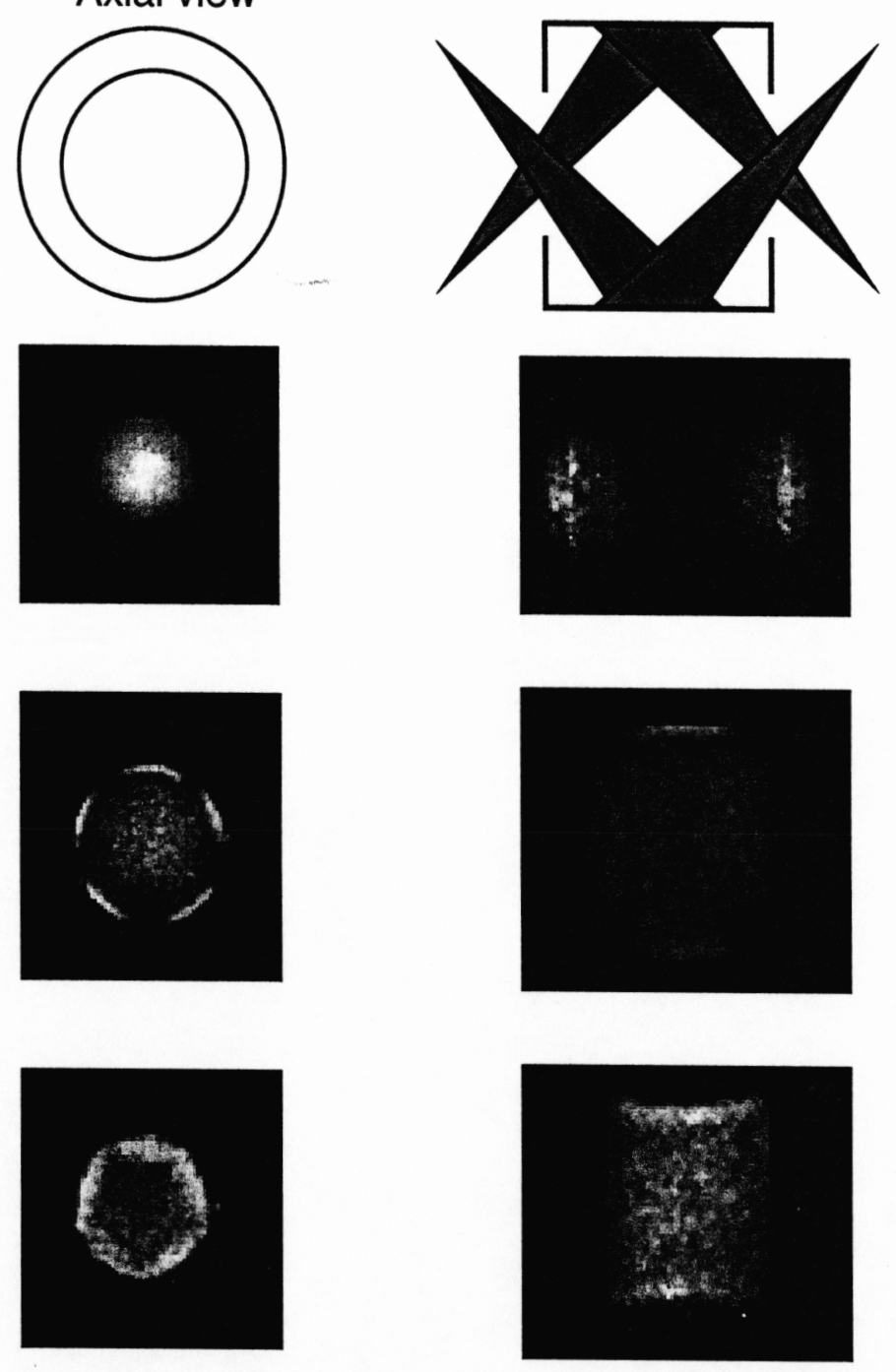

Fig.5

POP
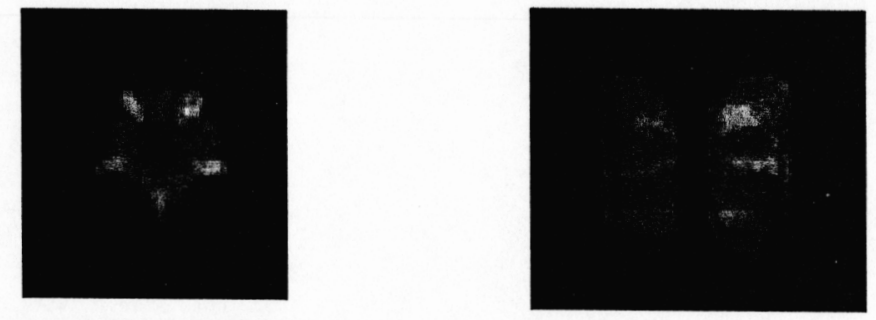

Back, et al. 

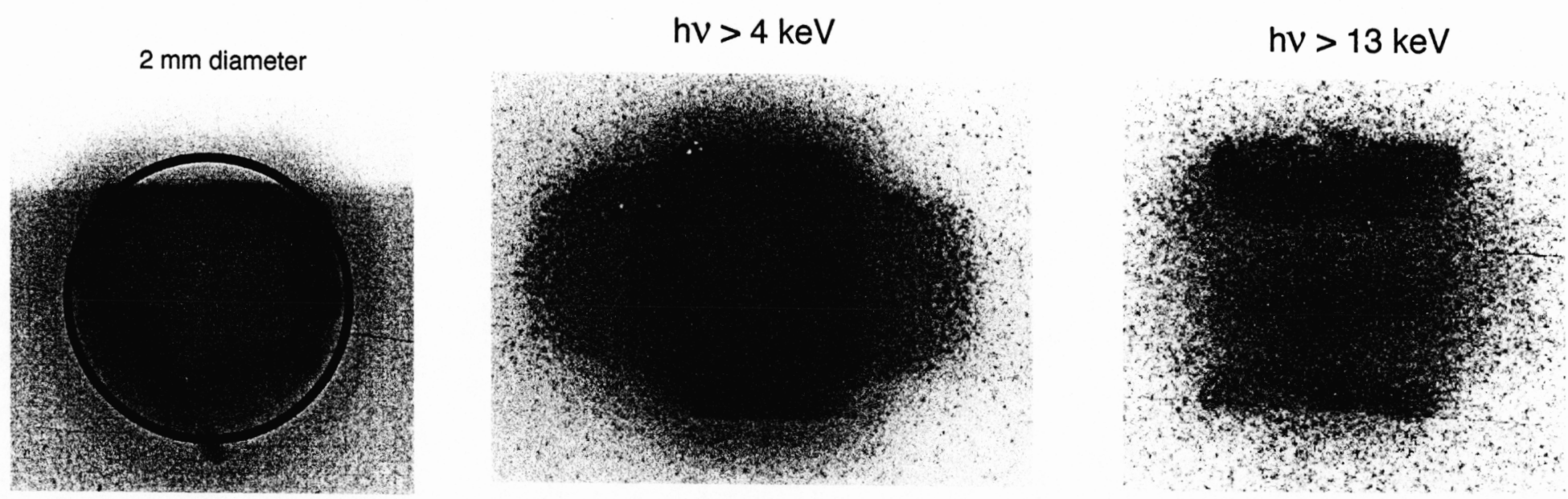

Fig.6

POP

Back, et al. 


$$
\text { at? }
$$




$$
\text { A. }
$$



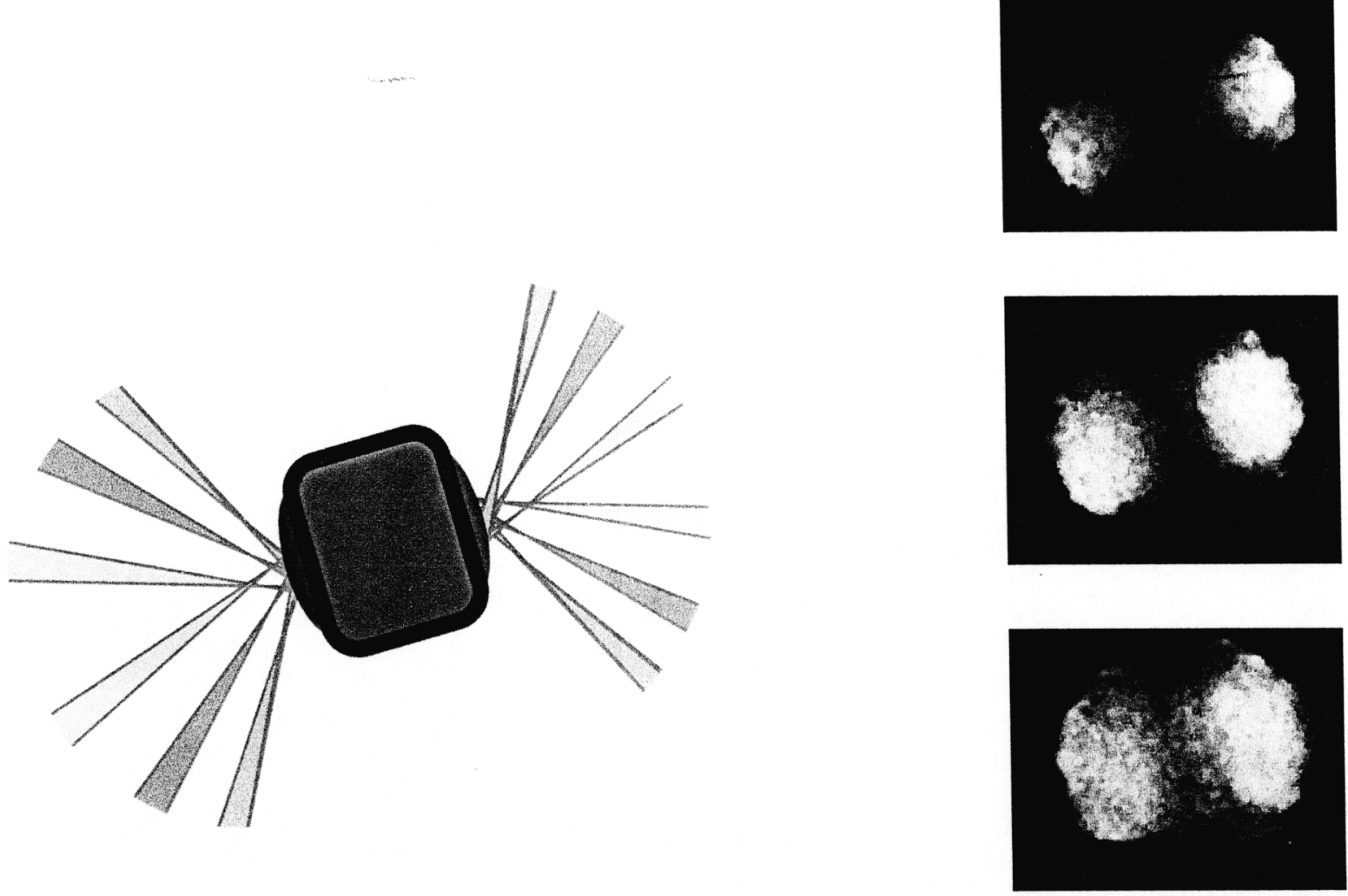

More imagese from 0.5 and $2.0 \mathrm{~atm}$ will be added

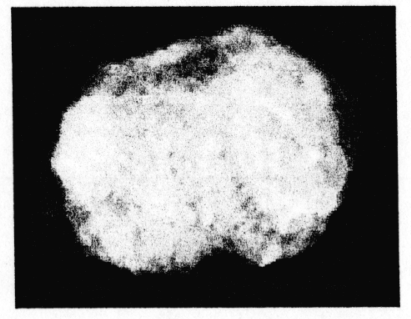

Fig.9

POP

Back, et al. 

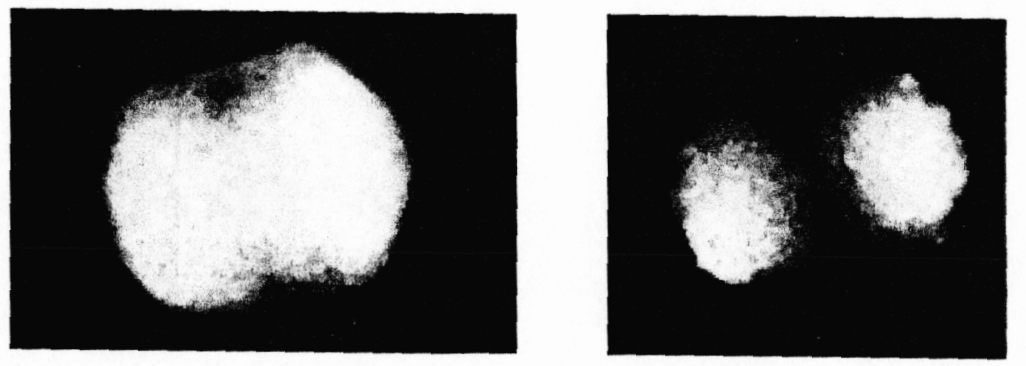

Fig. 10

POP

Back, et al. 


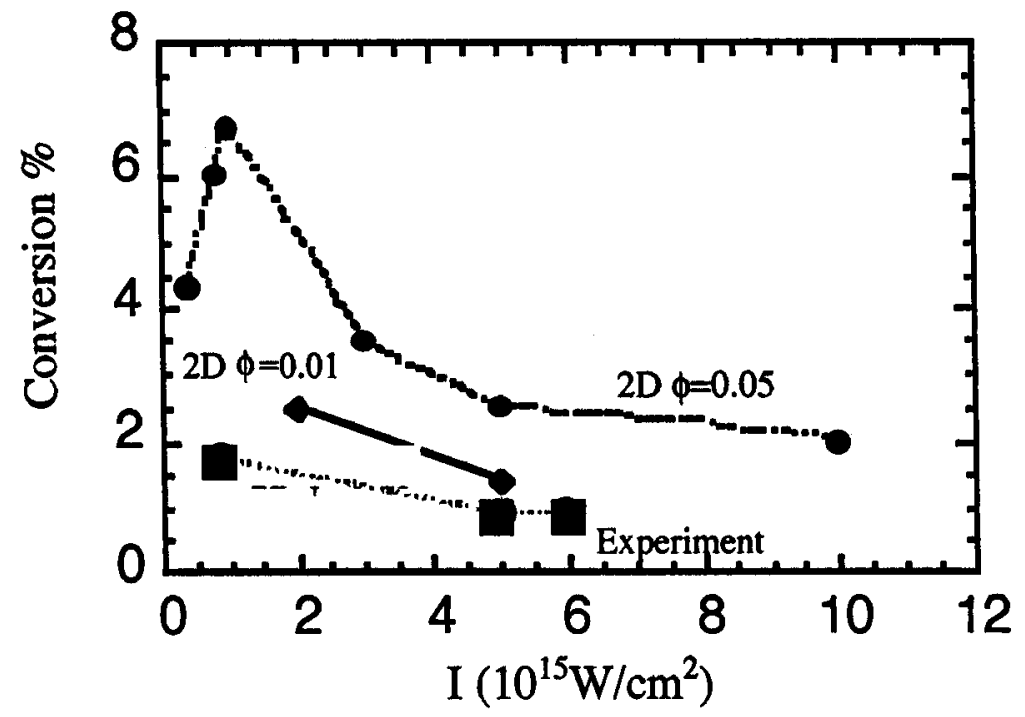

Fig.11

POP

Back, et al. 


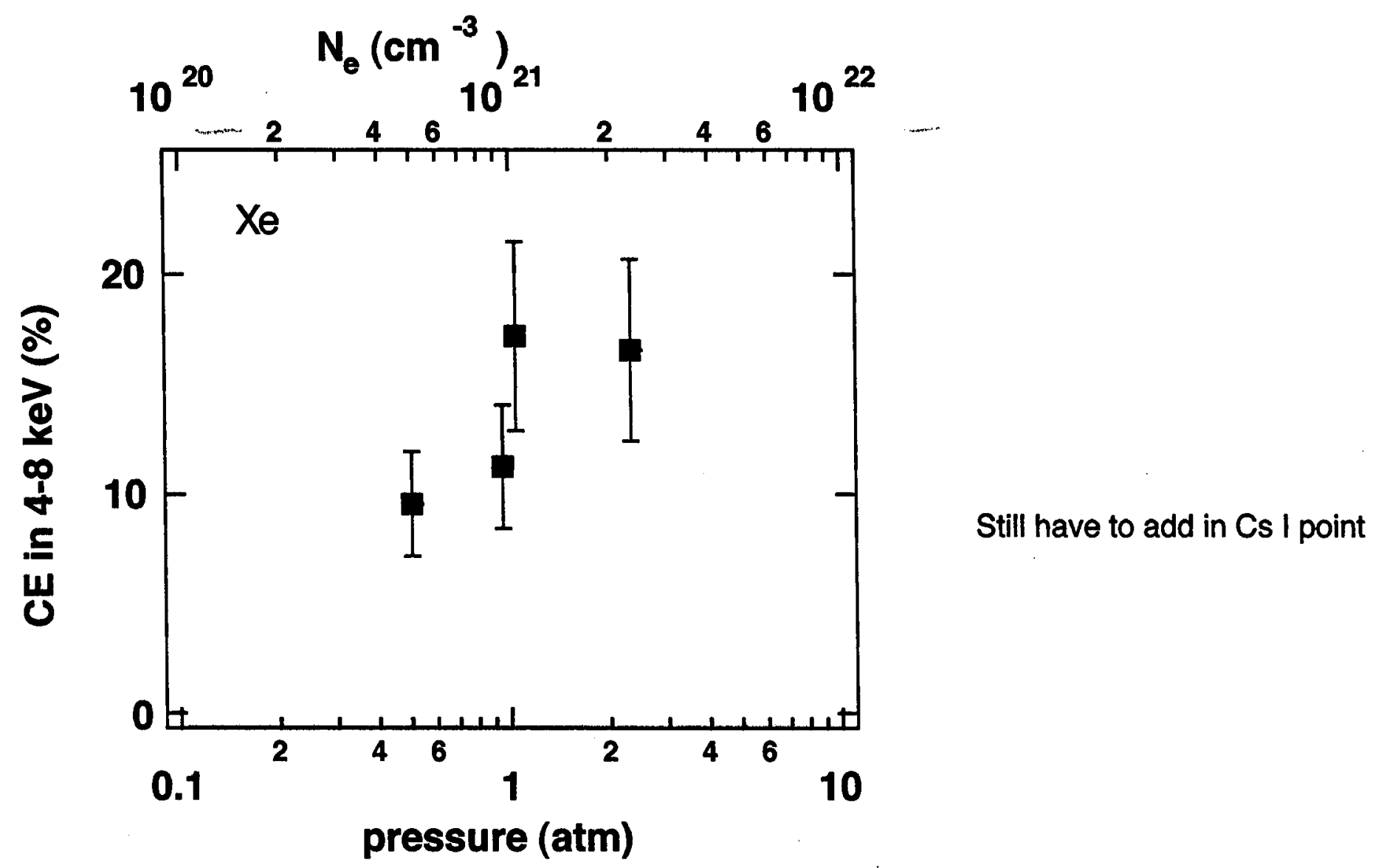

Fig.12

POP

Back, et al. 


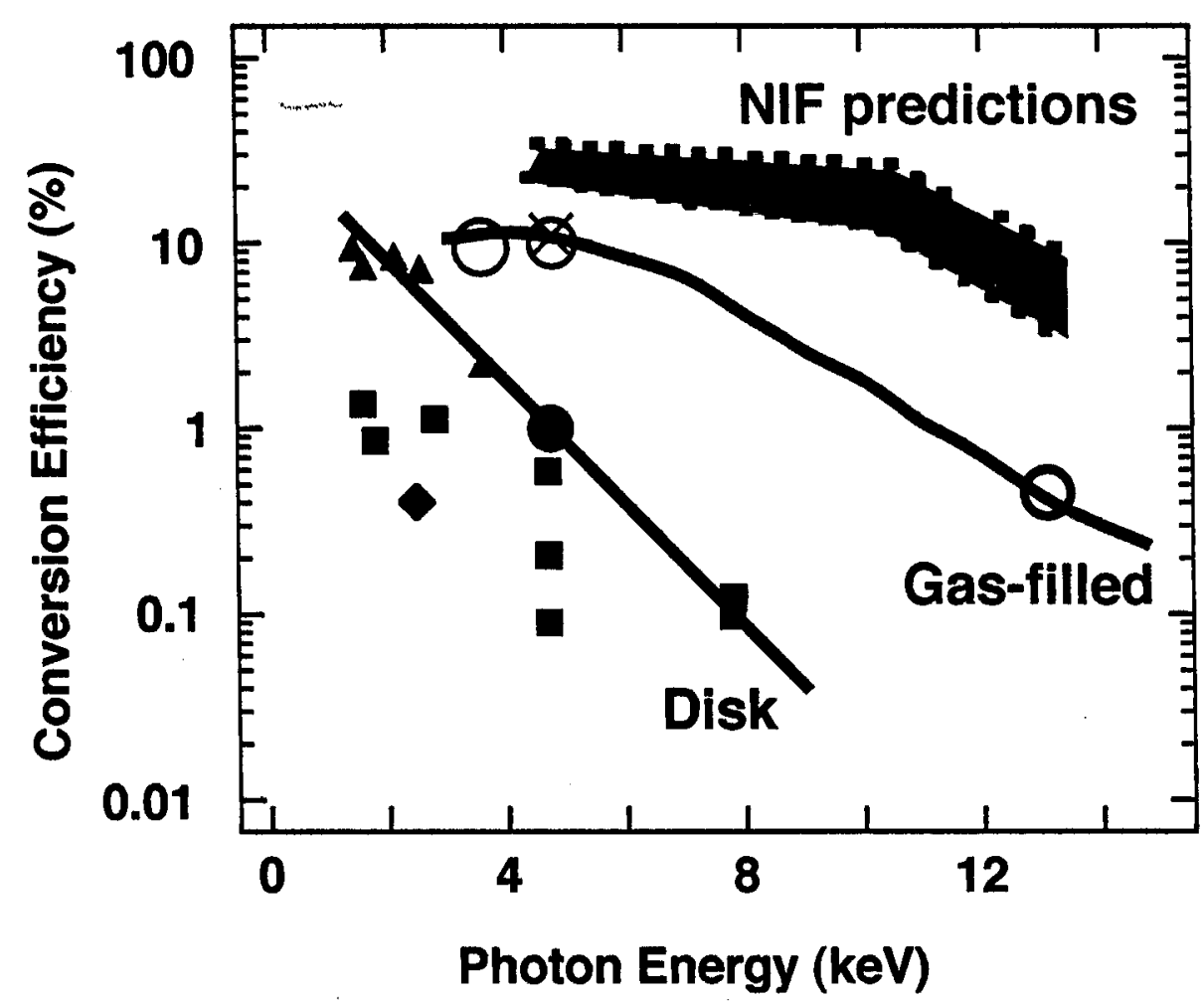

Fig.13 